\title{
Separating adverse-pressure-gradient and Reynolds-number effects in turbulent boundary layers
}

\author{
C. Sanmiguel Vila $\odot,{ }^{1}$ R. Vinuesa $\odot,{ }^{2}$ S. Discetti $\odot,{ }^{1}$ A. Ianiro $\odot,{ }^{1}$ P. Schlatter $\odot,{ }^{2}$ and R. Örlü $\odot^{2, *}$ \\ ${ }^{1}$ Aerospace Engineering Research Group, Universidad Carlos III de Madrid, 28911 Leganés, Spain \\ ${ }^{2}$ SimEx/FLOW, Engineering Mechanics, KTH Royal Institute of Technology, 10044 Stockholm, Sweden
}

(Received 15 October 2019; accepted 21 May 2020; published 17 June 2020)

\begin{abstract}
Zero pressure-gradient (ZPG) turbulent boundary layers (TBLs) at high Reynolds numbers (Re) and TBLs with adverse pressure gradients (APGs) share some similarities such as the emergence of an outer peak in the streamwise variance profile related to the enhancement of large-scale energy. Reynolds-number and pressure-gradient effects tend to cause such an energizing of the outer-layer structures, which makes it difficult to distinguish them, mainly because both effects are usually coupled. Using two experimental data sets of ZPG and APG TBLs in which Re and pressure gradient are analyzed independently, the present paper shows that the two effects have different imprints on the flow. In particular, the analysis shows that (1) the small-scale energy in APG TBLs is, contrary to canonical ZPG TBLs, not universal, but is dependent on the APG strength, (2) the APG enhances both the small- and large-scale energy in the outer region, and (3) the outer-peak location and the amplitude of the streamwise variance scale differently for increasing Re and APG magnitude. These findings may have ramifications on the development of turbulence models since the pressure gradient has a direct influence on the near-wall dynamics.
\end{abstract}

DOI: 10.1103/PhysRevFluids.5.064609

\section{INTRODUCTION}

There has been a continuous interest in the scaling of statistical quantities in wall-bounded turbulent flows. The knowledge of such scaling results, eventually, is necessary for the development of more refined turbulence models that work under varying conditions, and thus facilitate engineering design in general. However, extracting scaling trends is difficult, as one needs to rely on experiments (be it physical or numerical) that span an extended range of parameters such as the Reynolds number Re. In particular, the seminal work by Hutchins et al. [1], showcased how measurement-technique inefficiencies and uncertainties have blurred the scaling of the near-wall peak of the streamwise variance profile for decades. Since then growing evidence from high-Re numerical simulations and experiments in ZPG TBLs [2,3], but also pipe [4,5] and channels [6], has accumulated and established that the near-wall peak increases with increasing Reynolds number. A scale-decomposition analysis of the spectral contribution to the streamwise energy, as well as the concept of amplitude modulation of the small-scale fluctuations near the wall by large-scale outer-layer structures, indicated that this increase is related to the outer-layer structures which superpose their contribution down to the wall [7-11], thereby contributing to the otherwise universal, i.e.,

\footnotetext{
*Corresponding author: orlu@kth.se
}

Published by the American Physical Society under the terms of the Creative Commons Attribution 4.0 International license. Further distribution of this work must maintain attribution to the author(s) and the published article's title, journal citation, and DOI. Funded by Bibsam. 

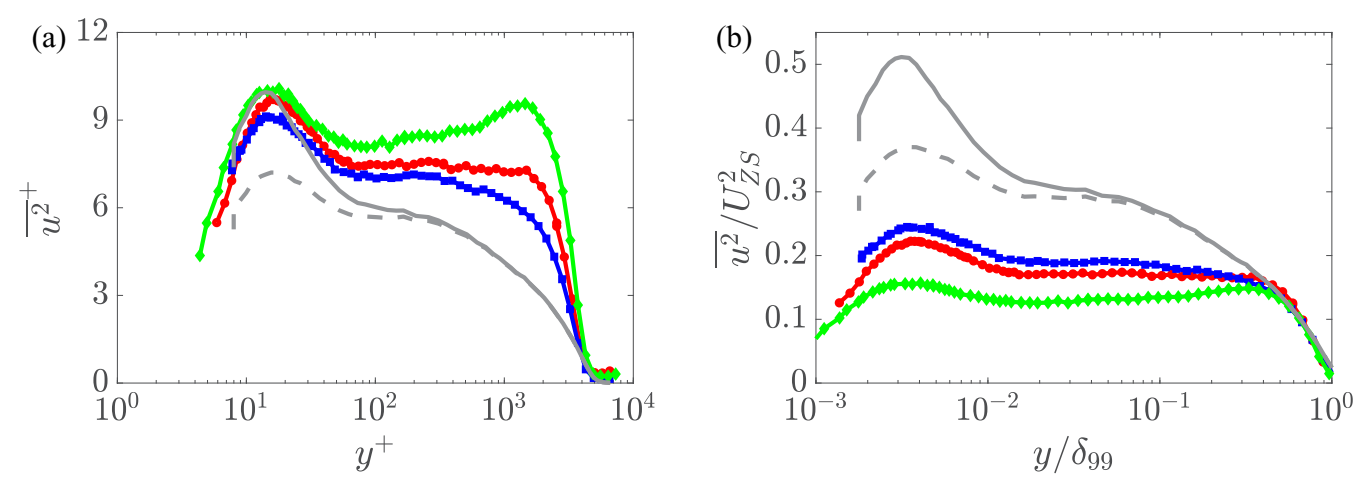

FIG. 1. Mean streamwise variance profiles at matched $\operatorname{Re}_{\tau} \approx 4400$, nondimensionalized by (a) the friction velocity $u_{\tau}$ and viscous length $\ell^{\star}$ and (b) the Zagarola-Smits (ZS) length scale $U_{\mathrm{ZS}}$ defined below and the boundary-layer thickness $\delta_{99}$. Colors represent $(-) \beta \approx 0$ (experimental data from Refs. [32,33]), ( $\square$ ) $\beta \approx 0.75,(\bigcirc) \beta \approx 1.1$, and $(\diamond) \beta \approx 2.2$. Gray dashed line indicates raw hot-wire data without applying the correction from Ref. [34].

Re-independent, small-scale energy. With the emergence of high-Re data, the controversy shifted towards a second, so-called outer peak in the streamwise variance profile [12], which previously was associated with insufficient spatial resolution, while more recent data support the existence of such a peak even if the final word is not spoken yet [13,14].

The increase of the near-wall peak as well as the emergence and growth of an outer peak in the streamwise variance profile is even more apparent for TBLs with an imposed adverse pressure gradient (APG) [15-18], even for considerably lower Re [19-23]. Not only due to this apparent similarity, but also because of the great engineering relevance of APG TBL flows, there has been a renewed interest in its assessment in terms of turbulence statistics [17,24-26]. The additional complication in the understanding of the scaling of turbulence statistics compared to ZPG TBL flows is that besides the Reynolds number, also the pressure-gradient strength and upstream history play a role [19]. Without anticipating too much from the results, this additional complication can be appreciated when considering the streamwise variance profiles at a fixed friction Reynolds number of $\operatorname{Re}_{\tau} \approx 4400\left(\operatorname{Re}_{\tau}=u_{\tau} \delta_{99} / v\right.$ where $u_{\tau}$ is the friction velocity, $\delta_{99}$ is the $99 \%$ of the boundary-layer thickness, and $v$ is the kinematic viscosity) in Fig. 1 for a ZPG and three different APG conditions, as expressed through the Rotta-Clauser pressure-gradient parameter $\beta$, which is defined as $\beta=\left(\delta^{*} / \tau_{w}\right)(d P / d x)$ (with $\delta^{*}$ denoting the displacement thickness, $\tau_{w}$ the mean wall-shear stress, and $d P / d x$ the pressure gradient along the streamwise coordinate). This figure highlights the similarities between Re and APG effects, such as increasing fluctuation amplitudes and the emergence of an outer peak [15], however, with differences in the scaling for the respective outer-peak locations [25,27].

Recent studies have both reinforced similarities but also identified differences between high-Re ZPG TBLs and APG TBLs, such as the work by Harun et al. [15], which calls for further experiments that allow for a differentiation between Re and APG effects. When it comes to the location of the outer peak [clearly emerging with increasing $\beta$ in Fig. 1(a)], the displacement thickness has been suggested to provide a Re- and APG-magnitude-independent scaling [25,27], clearly distinguishing it from the location of the corresponding peak in ZPG TBLs [10,27-29]. Another important aspect to assess the development of large-scale energy in the outer region at progressively higher $\mathrm{Re}$ is the emergence of a spectral outer peak, which in ZPG TBLs has a streamwise wavelength of around six boundary-layer thicknesses [30,31], but there are no conclusive statements in the literature regarding the power-spectral density distribution in the outer region of APGs. Furthermore, a number of studies have called for "high-quality near-wall data at high Reynolds numbers and covering a wide spectrum of pressure-gradient conditions" [27]. The present work aims at filling the identified gap 

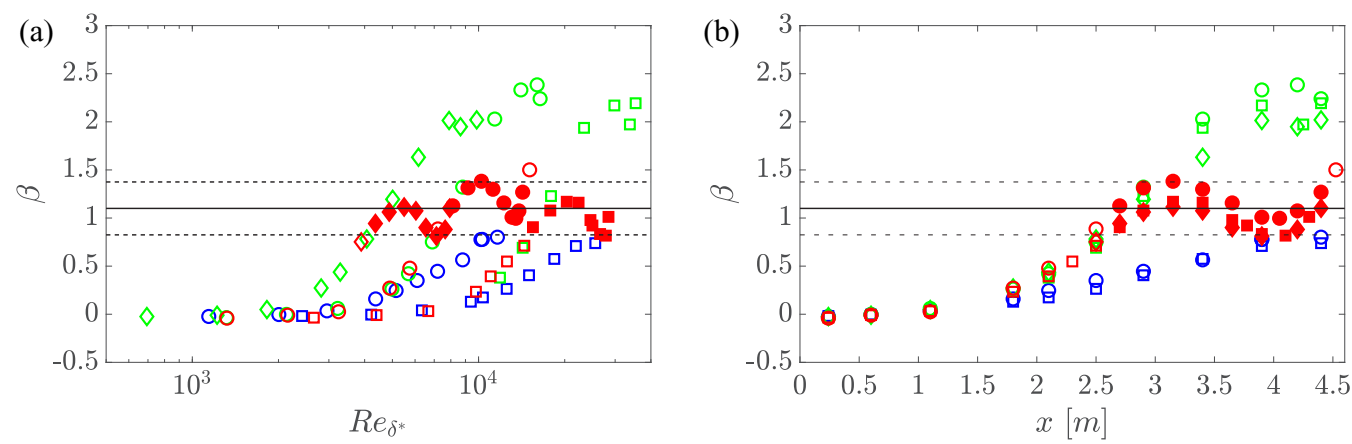

FIG. 2. Streamwise evolution of the Rotta-Clauser pressure-gradient parameter $\beta$ as function of the Reynolds number based on the displacement thickness $\operatorname{Re}_{\delta^{*}}$. The colors and symbols are listed in Table I. Reference black dash lines at $\beta=1.1 \pm 25 \%$ are included. Filled symbols indicated the region bounded by $25 \%$ deviation from $\beta \approx 1.1$, which henceforth will be denoted approximately constant $\beta$ region.

in terms of analyzing new APG TBL data that provide a wide range of Re and different APG conditions, including one case in which the pressure-gradient magnitude is approximately constant along its streamwise evolution in order to decouple Re from APG effects.

The paper is organized as follows. Section II describes the APG data set to be used throughout the paper. The spectral decomposition of the streamwise fluctuation energy in APG TBLs highlighting the difference to their ZPG counterpart is discussed in Sec. III. Upon introduction of a method to distinguish the outer-peak location in Sec. IV, the scaling of the inner and outer variance peak amplitude and location are presented in Sec. V, before concluding the work in Sec. VI.

\section{DESCRIPTION OF THE DATA SET}

The present analysis is based on the data sets from wind tunnel experiments of ZPG and APG TBL described in Refs. [32,33,35], which cover a range of $690<\operatorname{Re}_{\delta^{*}}<36000$, where $\operatorname{Re}_{\delta}^{*}$ denotes the Reynolds number based on the boundary-layer-edge velocity and displacement thickness $\delta^{*}$. The data sets were obtained with single hot-wire measurements performed in the Minimum Turbulence Level (MTL) wind tunnel at KTH Royal Institute of Technology. Details regarding the MTL wind tunnel in general and other details can be found in Refs. [33,35-37]. The current data sets consist of a ZPG configuration and three APG distributions. The pressure-gradient configurations were obtained by modifying the MTL roof geometry by means of wall inserts made of foam attached to the roof. The streamwise evolution of all the pressure-gradient configurations follow the same structure: an initial region where a favorable pressure gradient (FPG) is imposed, a second one where nearly ZPG conditions are established in order to obtain a well-behaved TBL [38], and finally an APG region which is the region of interest for the present investigation. The three APG configurations comprise a non-constant $\beta$ case with mild pressure gradient (maximum $\beta \approx 0.7$ ), a non-constant- $\beta$ case with a higher peak value of $\beta \approx 2.4$, and a case with an approximately constant value of $\beta \approx 1.1$. The resulting $\beta$ distributions are shown in Fig. 2. Table I reports a summary of the pressure-gradient distributions for the various cases under consideration.

An extensive description of the experimental setup, measurement technique and post-processing procedures, as well as a discussion of the data can be found in Refs. [32,33,35]. For the purpose of the present study, the boundary-layer parameters for the profiles employed in the first part of the study are reported in Table II. For the second part of this study, it is merely important to note that the viscous-scaled hot-wire length for all APG profiles is $\lesssim 30$, which ensures that the region where the outer peak is located is not biased through inadequate spatial resolution $[1,39]$. ZPG data from Ref. [32] are corrected using the correction proposed in Ref. [34] to rectify the attenuated 
TABLE I. Characteristic values for the different configurations in the APG experimental database [35]. Minimum and maximum $\beta$ values are reported in the approximately constant- $\beta$ region of configuration 1 given by $2.7 \mathrm{~m} \leqslant x \leqslant 4.4 \mathrm{~m}$ (Fig. 2 ).

\begin{tabular}{lcccccc}
\hline \hline Roof conf. & $U_{\text {ref }}[\mathrm{m} / \mathrm{s}]$ & Distribution type & Min. $\beta$ & Max. $\beta$ & Color & Symbol \\
\hline 1 & 6 & Approximately constant & 0.82 & 1.11 & Red & $\diamond$ \\
1 & 12 & Approximately constant & 1.00 & 1.38 & Red & $\bigcirc$ \\
1 & 30 & Approximately constant & 0.81 & 1.17 & Red & $\square$ \\
2 & 12 & Mildly increasing & 0.45 & 0.80 & Blue & $\bigcirc$ \\
2 & 30 & Mildly increasing & 0.41 & 0.74 & Blue & $\square$ \\
3 & 6 & Strongly increasing & 1.20 & 2.12 & Green & $\diamond$ \\
3 & 12 & Strongly increasing & 1.32 & 2.38 & Green & $\bigcirc$ \\
3 & 30 & Strongly increasing & 1.23 & 2.19 & Green & $\square$ \\
\hline \hline
\end{tabular}

inner peak observed at the friction Reynolds number of $\operatorname{Re}_{\tau} \approx 4400$ (Figs. 1 and 4 ) due to the large inner-scaled hot-wire length $L^{+}$(cf. Table II).

The friction velocity $u_{\tau}$, which is used for the inner normalization, is calculated by means of the composite profile [40]. To obtain friction velocities that are independent of the log-law constants (inherent in the composite profile), the fit of the measured streamwise mean velocity profile to the composite profile was limited to $y^{+}<12$ wherever possible. Note, however, that some of the measurement stations were also validated against independent wall-shear-stress measurements by means of oil-film interferometry. The estimation of the boundary-layer thickness $\delta_{99}$ and $U_{\text {edge }}$ was performed according to the procedure reported in Ref. [41], which is based on the diagnostic-plot concept [12]. Using the value of $\delta_{99}$ as the upper limit of integration, $\delta^{*}$ and $\theta$ are obtained. With these quantities, the shape factor $H_{12}$ and Rotta-Clauser pressure-gradient parameter $\beta$ are calculated, where the latter is defined as $\beta=\left(\delta^{*} / \tau_{w}\right)(\mathrm{d} P / \mathrm{d} x)$, where $\tau_{w}$ is the mean wall-shear stress and $d P / d x$ is the pressure gradient along the streamwise coordinate.

\section{SCALE RELATIONSHIP}

In order to reveal the pressure-gradient effects on the scale organization, Fig. 3 shows the premultiplied streamwise energy spectra $\left(\kappa_{x}^{+} \Phi_{u u}^{+}\right)$plotted against the inner-scaled wall-normal distance $y^{+}$and the streamwise wavelength $\lambda_{x}^{+}$. From the observation of the results of Ref. [35], where the premultiplied streamwise energy spectra were analyzed in terms of the inner-scaled time $t^{+}$, it is apparent that a cutoff based only on a fixed $t^{+}$value would not completely separate the small and large scales in the spectra. For this reason, Taylor's frozen turbulence hypothesis is used to analyze $\kappa_{x}^{+} \Phi_{u u}^{+}$in order to be able to define a proper scale-cutoff wavelength in the high-Re spectra. With this purpose in mind, the convection velocity, $U_{c}$, has been taken as the local mean velocity, except for the region $U_{c}^{+}<10$ where it has been set to $10 u_{\tau}$ following Ref. [42]. This estimation of $U_{c}$ is in agreement with literature studies focusing on the amplitude modulation in

TABLE II. Boundary-layer parameters for the profiles extracted from the experimental database of Refs. [32,33,35].

\begin{tabular}{lccccccccc}
\hline \hline Configuration & $\beta$ & $U_{\text {edge }}[\mathrm{m} / \mathrm{s}]$ & $\operatorname{Re}_{\delta^{*}}$ & $\operatorname{Re}_{\theta}$ & $\operatorname{Re}_{\tau}$ & $\delta_{99}[\mathrm{~mm}]$ & $H_{12}$ & $L^{+}$ & Data set \\
\hline 1 & 1.12 & 24.4 & 28440 & 19780 & 4300 & 90.7 & 1.44 & 13 & {$[35]$} \\
2 & 0.71 & 27.0 & 25570 & 18120 & 4190 & 76.5 & 1.41 & 29 & {$[35]$} \\
3 & 2.22 & 23.2 & 35370 & 23450 & 4410 & 101.6 & 1.51 & 23 & {$[35]$} \\
ZPG & $\approx 0$ & 40.03 & 19670 & 15040 & 4480 & 49.7 & 1.31 & 50 & {$[32],[33]$} \\
\hline \hline
\end{tabular}



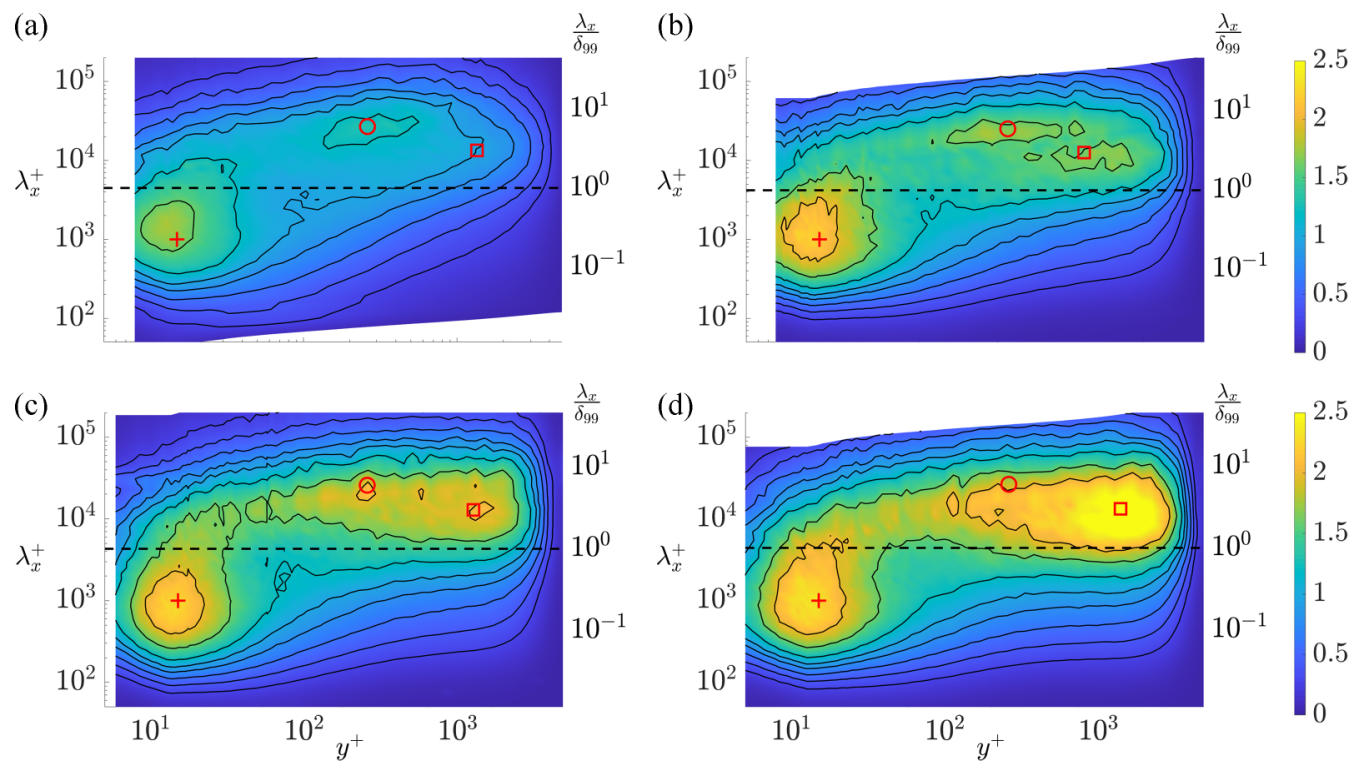

FIG. 3. Inner-scaled premultiplied energy spectra of the streamwise velocity at $\operatorname{Re}_{\tau} \approx 4400$ with contour levels at $\kappa_{x}^{+} \Phi_{u u}^{+}=0.25,0.4,0.575,0.775,0.95,1.2,1.6,2.0$, for pressure-gradient strengths (a) $\beta \approx 0$ (experimental data from Ref. [32]), (b) $\beta \approx 0.75$, (c) $\beta \approx 1.1$, (d) $\beta \approx 2.2$. Auxiliary red symbols are located at the following coordinates: $(+)\left(y^{+}=15, \lambda_{x}^{+}=1000\right),(0)\left(y^{+}=3.9 \mathrm{Re}_{\tau}^{0.5}, \lambda_{x} / \delta_{99}=6\right)$ for all the cases. Additionally red $(\square)$ symbols are located at $\left(y^{+}=y_{\max , \text { outer }}^{+}, \lambda_{x} / \delta_{99}=3\right)$ for all the APG cases and $\left(y / \delta_{99}=0.3, \lambda_{x} / \delta_{99}=3\right)$ for the ZPG case. An auxiliary horizontal dashed line is placed at $\lambda / \delta_{99}=1$.

APG TBLs $[15,43,44]$. Figure 3 shows that the Reynolds number is high enough that an outer spectral peak is present in the APG TBLs at a wavelength $\lambda_{x} \approx 6 \delta_{99}(0)$, as observed also for ZPG TBLs [9]. Furthermore, we observe the emergence of a second spectral peak, ascribed to APG effects, with wavelength $\lambda_{x} \approx 3 \delta_{99}(\square)$, a result that is in agreement with previous experiments
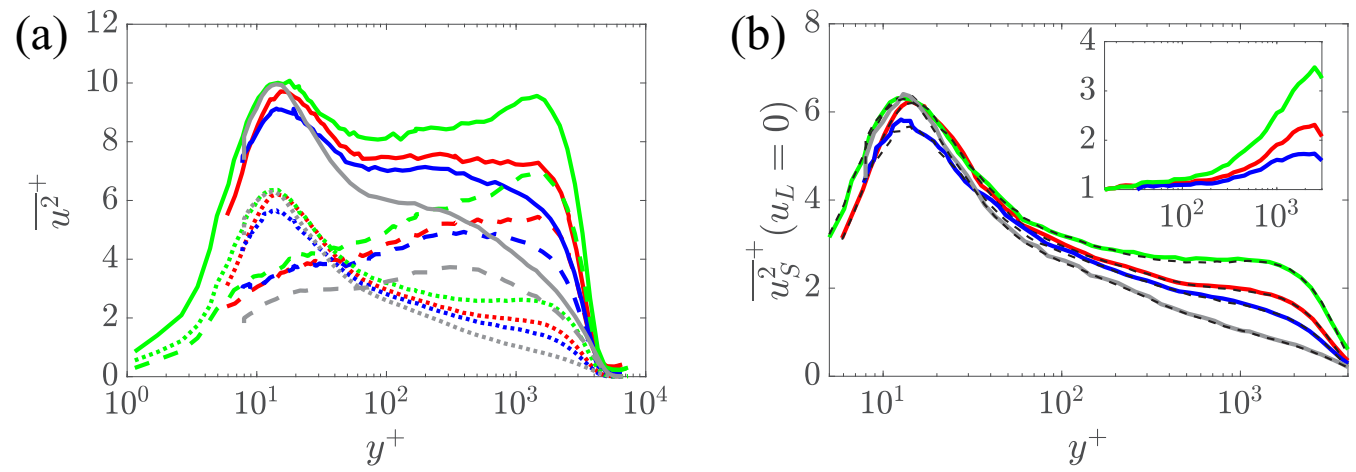

FIG. 4. (a) Decomposition of the velocity fluctuations (solid lines) into a small-scale (dotted lines) and a large-scale (dashed lines) component for $\operatorname{Re}_{\tau} \approx 4400$. (b) Small-scale variance conditioned on a large-scale fluctuation value of $u_{L}^{+}=0,{\overline{u_{S}^{2}}}^{+}\left(u_{L}=0\right)$, for $\operatorname{Re}_{\tau} \approx 4400$ together with the small-scale (dotted lines) component from (a). The inset represents the ratio between the APG cases and the ZPG case, i.e., ${\overline{u_{S}^{2}}}^{+}\left(u_{L}=0\right)_{\mathrm{APG}} /{\overline{u_{S}^{2}}}^{+}\left(u_{L}=0\right)_{\mathrm{ZPG}}$. Gray $(-)$ represents $\beta \approx 0$, blue $(-) \beta \approx 0.75$, red $(-) \beta \approx 1.1$, and green $(-) \beta \approx 2.2$. 
such as those reported in Ref. [15]. The $y^{+}$location of the APG-related spectral peak is identified according to the method proposed in Sec. IV.

The effect of the interaction between the different scales and their effect on the Reynolds stresses is quantified via a scale-decomposition analysis $[15,44]$. Based on previous results $[15,44,45]$, the cutoff wavelength has been applied at $\lambda_{x} / \delta_{99}=1$, which is a reasonable compromise to separate the inner and outer peaks in the spectra, as apparent from the horizontal dashed line which is represented in the spatial spectral maps shown in Fig. 3. Although the use of Taylor's hypothesis to define the streamwise wavelength depends on the choice of the convection velocity throughout the boundary layer, this is the only possibility in single-point hot-wire measurements. Nonetheless, using the local velocity as a surrogate for the convection velocity is a widely accepted and utilized method [9,42,46], valid according to Ref. [15] as long as one considers the region beyond $U^{+} \gtrsim 10$, which is the focus of the present investigation.

Figure 4(a) shows the small- and large-scale components of the streamwise velocity fluctuations, decomposed by the aforementioned scale decomposition. It is observed that with increasing pressure-gradient strength the penetration of the large-scale components toward the near-wall region increases, as is also the case for ZPG TBLs with increasing Re. Previous studies on ZPG TBLs have established that the small-scale energy is universal throughout the entire boundary-layer thickness (in inner-scaling) [47], such as shown in Fig. 1 in Ref. [48] or Fig. 6 in Ref. [10]. This "well accepted argument that the viscous-scaled small-scale turbulence energy is invariant with Reynolds number" [49], however, does clearly not extend to APG cases discussed here, since the small-scale energy contribution is enhanced in the outer region (where spatial resolution effects are not to be expected [1]). The amplitude of the small-scale energy contribution for the inner layer, on the other hand, agrees also quantitatively with previous studies $[10,48]$. The profiles shown here are collapsing with the profiles from Refs. [10,48] in the inner and logarithmic region, being appreciated only differences in the final part of the outer region due to the different Reynolds number of the reported studies. This finding has an important implication on methods, models or theories that are based on small-scale universality throughout the boundary layer. For instance, hot-wire spatial-resolution correction schemes that are based on some universal small-scale energy contribution assume that "attenuation due to insufficient spatial resolution is largely restricted to the viscous-scales" [50] and they exploit the "invariance of inner-scaled small-scale contribution with Reynolds number ... to apply the missing energy obtained from a lower Reynolds number DNS data set to higher Reynolds number flows" [48]. Similarly, corrections for the influence of calibration misalignment for cross-wire probes have also relied "on the premise that the viscousscaled small-scale contributions to the turbulence stresses is invariant with Re and solely dependent on the viscous-scaled spatial resolution of the probe" [51]. Such correction schemes should thus be employed with caution for APG TBLs or any other TBL flow that exhibits a different scale decomposition, e.g., due to application of flow control schemes or surface roughness. Moreover, this fact also implies that comparisons at (relatively large and) matched viscous-scaled hot-wire lengths [15] will not ensure the absence of measurement bias due to spatial-resolution effects. In this respect, it should be noted that for the present work the correction proposed by Smits et al. [34] has only been employed on the ZPG TBLs profiles, in order not to introduce any bias in the APG data set. Similar concerns might be raised for correction methods for spatial resolution in particle image velocimetry (PIV) [52].

In order to isolate the pressure-gradient effect in the small-scale organization, the methodology described in Ganapathisubramani et al. [53] and Yoon et al. [54] is employed to calculate the smallscale energy of the streamwise velocity fluctuations $\left(u_{S}^{2}\right)$ conditionally averaged for a value of the large-scale fluctuations $\left(u_{L}\right)$ equal to 0 . This represents the small-scale behavior when the influence of the large scales is weak. Figure 4(b) shows $\overline{u_{S}^{2}}\left(u_{L}=0\right)$ profiles at nearly equivalent $\operatorname{Re}_{\tau}$ for the different $\beta$ conditions, compared to the (unconditioned) small-scale component. The fact that both the unconditioned and conditioned small-scale energies overlap is expected due to the symmetric character of the large-scale signal. In this figure it can be appreciated that the small-scale variance 


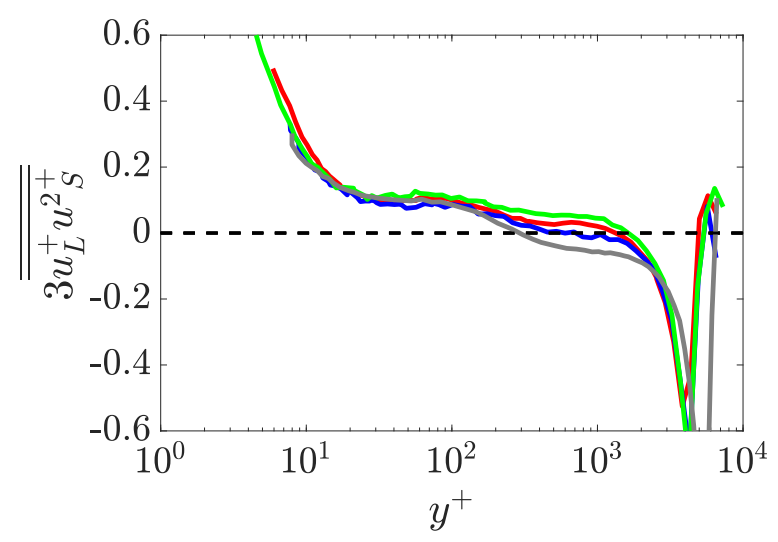

FIG. 5. Dominant scale-decomposed skewness term, $3 \overline{\overline{u_{L}^{+} u_{S}^{2+}}}$ for $\operatorname{Re}_{\tau} \approx 4400$ (right). Gray (-) represents $\beta \approx 0$, blue (-) $\beta \approx 0.75$, red $(-) \beta \approx 1.1$, and green $(-) \beta \approx 2.2$.

is increased with increasing $\beta$ in the outer region. In the near-wall region, this quantity exhibits similar near-wall peaks in both ZPG and APG cases, indicating that the higher inner peak observed in the streamwise variance with increasing $\beta$ is a consequence of the footprint of the large-scale motions. When comparing the ${\overline{u_{S}^{2}}}^{+}\left(u_{L}=0\right)$ profile at higher $y^{+}$, the effect of the pressure gradient on the small-scales starts to become apparent with increasing ${\overline{u_{S}^{2}}}^{+}$values within the logarithmic region. This shows that small-scale activity is increased not only by the footprint of the large scales. This observation could be associated with a displacement of the small-scale activity from the nearwall region towards the outer part of the boundary layer. This is in agreement with the results in Ref. [17], who reported that the sweep/ejection events of the TBL are displaced farther from the wall with increasing strength of the pressure gradient. Similarly, the results are also in accordance with those in Ref. [23], who reported increased wall-normal convection in APG TBLs. These results have important implications, since they challenge the concept that the small-scale contribution is universal and that Reynolds-number and pressure-gradient effects express themselves only in the large scales. It has has, e.g., been shown for ZPG TBLs that "the only thing that changes as Reynolds number increases is the large-scale energy" [50]. Instead, here, we show that both Re and APG effects contribute to the small-scale energy in the outer-layer.

\section{LOCATION OF THE OUTER PEAK}

The increase of the small-scale activity in the outer part of the boundary layer might raise a question about modulation effects throughout the boundary layer. In order to understand the interaction between the small and large scales, the amplitude modulation, which is defined as the modulation of the small-scale fluctuations by the large scales in the flow is analyzed in Fig. 5. This is done using the correlation $3 \overline{\overline{u_{L}^{+} u_{S}^{2+}}}$ [where $\overline{\bar{X}}=\bar{X} /\left(\overline{u^{+^{2}}}\right)^{3 / 2}$ for any variable $X$ ] [55]. This quantity is described in studies such as Refs. [45,55] and is found to be the only Reynolds-number-dependent component of the scale-decomposed skewness factor. The results show a highly modulated near-wall region. In general, the modulation in the logarithmic and outer region $\left(y^{+}>100\right)$ is higher in the APG cases with increasing $\beta$, as a result of the enhancement of the large-scale organization in these flows. Further away from the near-wall region, the modulation decreases until becoming negative in the middle of the log-region for the ZPG case and at a location closer to the outer peak position in the APG cases. This may indicate that the observed outer spectral peak (cf. Fig. 3) is a mixed effect of large-scale events with the small-scales ejected from the inner region [17,23]. 

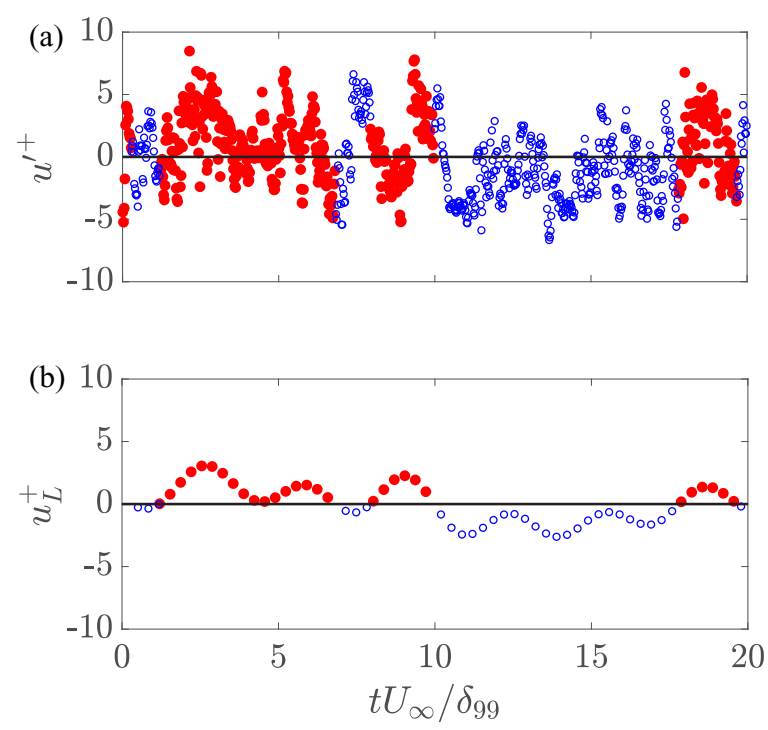

FIG. 6. (a) Instantaneous example of fluctuating signal $\left(u^{\prime+}\right)$ and (b) large-scale fluctuations component $\left(u_{L}^{+}\right)$computed based on a filter timescale of $t U_{c} / \delta_{99}=1$ at $y^{+} \approx 15$. Example case at $\operatorname{Re}_{\tau} \approx 4400$ and pressure-gradient strength $\beta \approx 1$.1. Red filled symbols represent signal segments corresponding to $u_{L}^{+}>0$ and blue empty symbols to $u_{L}^{+}<0$.

The method proposed herein for the identification of the outer-peak position is based on the interaction between small and large scales, and does not rely on geometrical constraints, in contrast to Ref. [3], therefore being valid even for APG TBL flows with mild values of $\beta$. Our method builds upon the cross-over of the streamwise fluctuation variance profile conditioned to high- and low-momentum events. The hypothesis behind this is that such a cross-over point indicates the regions where either the shear directly caused by the wall or the local shear induced by the external pressure gradient, respectively, are dominant in their effect on the streamwise velocity fluctuation variance. The first step of the method is to discriminate between events related to high- and lowspeed fluctuations. To this end, the large-scale component $\left(u_{L}^{+}\right)$is used as a filtering criterion to determine which raw-signal segments are related with events $u_{L}^{+}>0$ or $u_{L}^{+}<0$. This is shown in Fig. 6, where an instantaneous fluctuating velocity sample, $u^{\prime+}$ and its associated $u_{L}^{+}$are presented. The streamwise variance $\left.\overline{u^{2+}}\right|_{u_{L}^{+}>0}$ conditioned on $u_{L}^{+}>0$ can be defined as

$$
\left.\overline{u^{2^{+}}}\right|_{u_{L}^{+}>0}=\frac{\left.\sum u^{2^{+}}\left(u_{L}^{+}>0\right)\right|_{u_{L}^{+}(y)}}{N\left[u^{+}\left(u_{L}^{+}>0\right)\right]} .
$$

From the segments with events $u_{L}^{+}<0$, the $u_{L}^{+}<0$ variance $\left.\overline{u^{2+}}\right|_{u_{L}^{+}<0}$ can also be obtained. Figure 7 shows the resulting variance profiles and their difference. The results indicate that the point of intersection of the $\left.\overline{u^{2+}}\right|_{u_{L}^{+}>0}$ and $\left.\overline{u^{2+}}\right|_{u_{L}^{+}<0}$ curves, which is shown in Fig. 7, corresponds to the location of the outer peak due to the pressure-gradient effects in APG TBLs, i.e., $y_{\text {max,outer }}$. This is observed in all the profiles from the present study, as these locations are indicated in the spectral maps in Fig. 3. Figure 7(a) shows that the variance conditioned on low-speed events $u_{L}^{+}<0$ is nearly constant in the region $y^{+}>100$, while the variance due to high-speed events $u_{L}^{+}>0$ decreases throughout the whole logarithmic region. Figure 7(b) shows the quantity $\left.\overline{u^{2+}}\right|_{u_{L}^{+}>0}-\left.\overline{u^{2+}}\right|_{u_{L}^{+}<0}$, which resembles the shape of the modulation $3 \overline{\overline{u_{L}^{+} u^{2+}}}$ represented previously in Fig. 5; nonetheless, it is 

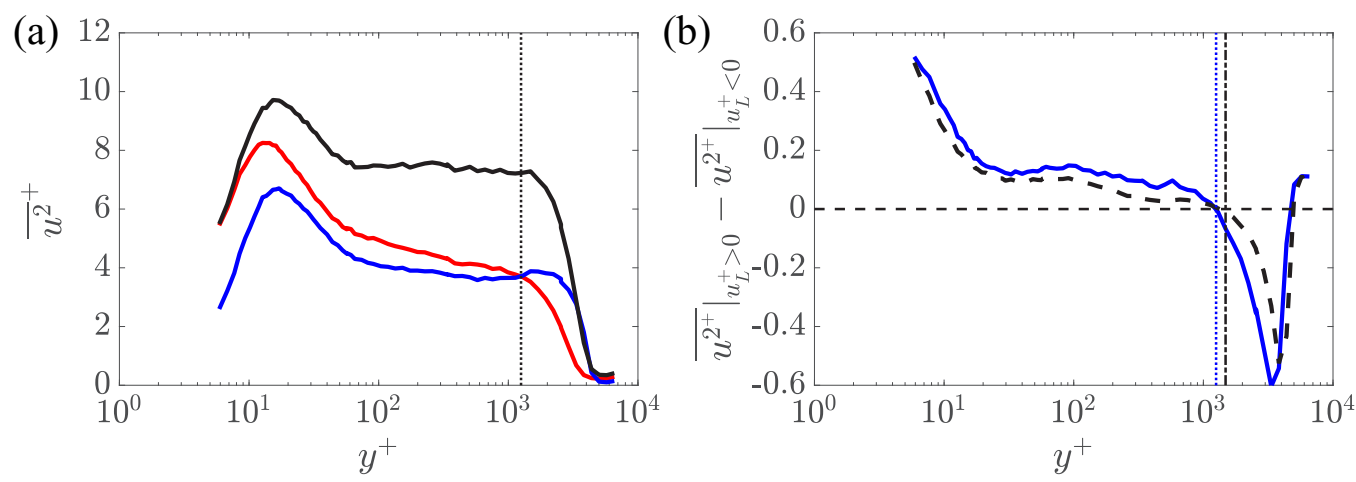

FIG. 7. (a) Streamwise variance calculated by signal segments with positive large-scale fluctuations $\left.\overline{u^{2+}}\right|_{u_{l}^{+}>0}$ (red), negative large-scale fluctuations $\left.\overline{u^{2+}}\right|_{u_{L}^{+}<0}$ (blue), and with the full signal length $\overline{u^{2+}}$ (black). (b) Difference between the streamwise variance calculated by signal segments with positive and negative large-scale fluctuations $\left.\overline{u^{2+}}\right|_{u_{L}^{+}>0}-\left.\overline{u^{2^{+}}}\right|_{u_{L}^{+}<0}$ (black dashed line) and dominant scale-decomposed skewness term, $3 \overline{\overline{u_{L}^{+} u_{S}^{2+}}}$ (blue line). Example case at $\operatorname{Re}_{\tau} \approx 4400$ and pressure-gradient strength $\beta \approx 1.1$. The dashed vertical lines indicate approximately the cross-over point between the $\left.\overline{u^{2+}}\right|_{u_{L}^{+}>0}$ and $\left.\overline{u^{2+}}\right|_{u_{L}^{+}<0}$ curves, i.e., $\left.\overline{u^{2+}}\right|_{u_{L}^{+}>0}-\left.\overline{u^{2+}}\right|_{u_{L}^{+}<0}=0$.

important to remark that these quantities are not identical, thus, their zero-crossing points are not the same as it is highlighted here.

The method set out in this section has been tested and validated in all the cases from the database presented in Ref. [35]. The locations of the $y_{\max \text {,outer }}$ obtained with the present method have also been verified using the outer spectral peak obtained when representing the premultiplied spectral map as in Fig. 3. Apart from that, since our method is only conditioned to high- and low-momentum events, it can be also applied to ZPG TBLs. As a consequence, the present methodology is also employed to locate $y_{\max , \text { outer }}$ in the experimental ZPG cases used in this study. The results of the $y_{\max , \text { outer }}$ obtained for ZPG TBLs follow the expected relation 3.9Re $\tau_{\tau}^{0.5}$ from Ref. [9].

\section{SCALING OF VARIANCE PEAKS}

To further reveal the differences between the outer-peak scaling behavior of ZPG and APG TBLs, in this section a comparison of the Re evolution for both peaks and an assessment of the $\beta$ evolution for the APG TBL outer peak is performed. This analysis aims to complement the study performed by Maciel et al. [27] in which the APG TBL peak evolution (of the streamwise variance profile) is investigated in the low-Re and high- $\beta$ range, without differentiation between the inner and outer peaks. In this study, the outer peak location $y_{\max , \text { outer }}$ and its corresponding amplitude $\overline{u^{2}}$ are located using the criterion described in Sec. IV. Hence, it is important to note that the maxima in $\bar{u}^{2}$ from the inner and outer regions are analyzed independently. In the following, the ZPG outer-peak location and its streamwise-variance value have been calculated in the range $1800<\operatorname{Re}_{\tau}<5500$ using the method outlined in Sec. IV in order to be able to compare both outer peak results. Inner peak results for the ZPG cases are not shown, due to the above-mentioned attenuation issues.

Figure 8 shows the outer-scaled evolution of the near-wall and outer-peak values of the streamwise variance and the location of the outer peak for the approximately constant- $\beta$ region from configuration 1. Figure 8(a) presents the evolution of the near-wall and outer peaks using the Zagarola-Smits velocity $U_{\mathrm{ZS}}=U_{\infty} \delta^{*} / \delta_{99}, \overline{u^{2}} / U_{\mathrm{ZS}}^{2}$; after a few points the values start following a slowly increasing trend with increasing $\operatorname{Re}_{\delta^{*}}$ for the ZPG case. In this respect, it is interesting to note that $\overline{u^{2}} / U_{\mathrm{ZS}}^{2}$ increases with increasing Re, while the APG data remain roughly constant. 

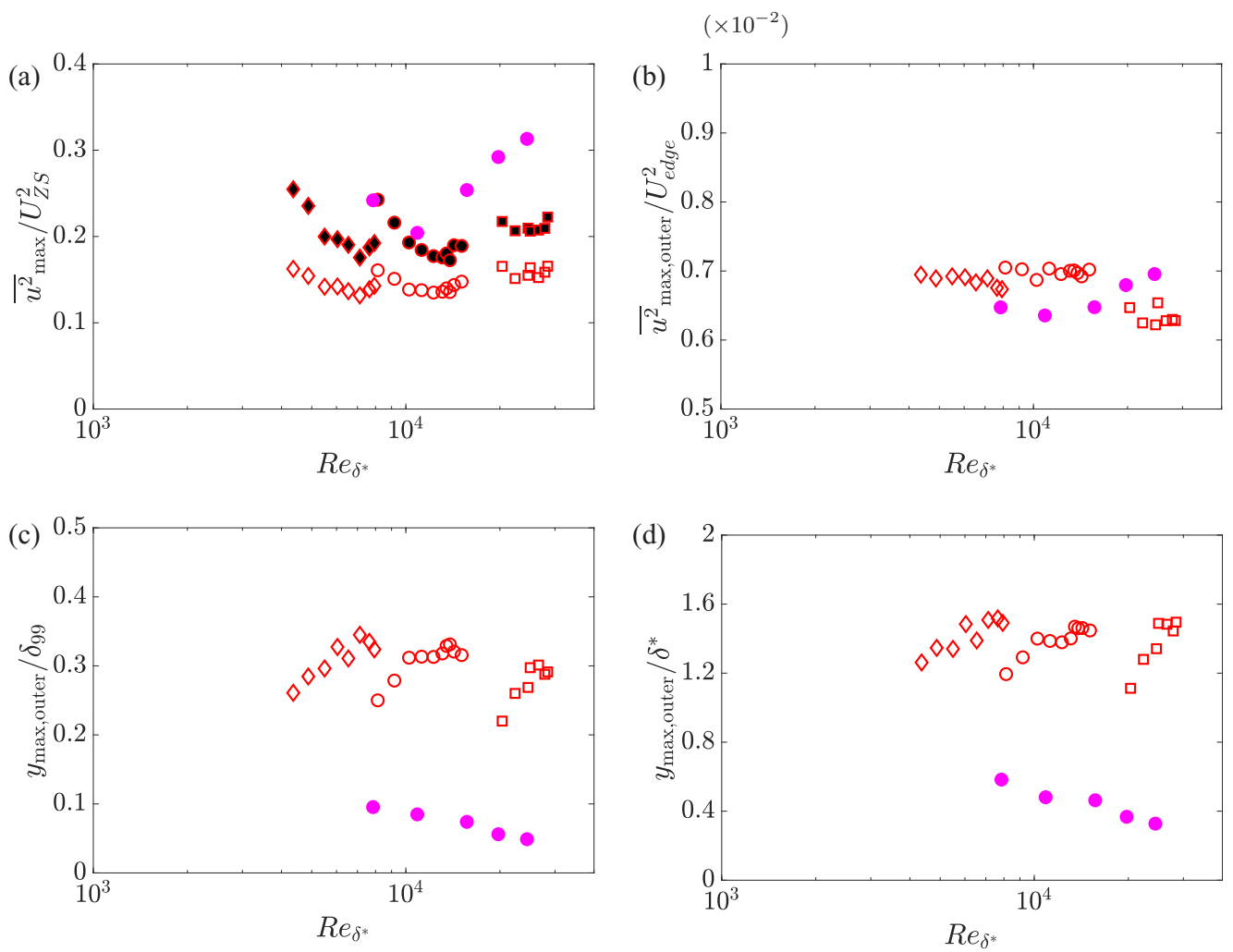

FIG. 8. Maximum value of the streamwise variance normalized with (a) $U_{\mathrm{ZS}}$ and (b) $U_{\text {edge }}$ as a function of $\operatorname{Re}_{\delta^{*}}$. Black filled symbols represent the near-wall peak and open symbols the outer peak. Magenta symbols indicate the outer peak values for the ZPG cases from Ref. [32]. Wall-normal position of the outer-peak location normalized with (c) $\delta_{99}$ and (d) $\delta^{*}$ as a function of $\operatorname{Re}_{\tau}$. The data correspond to the approximately constant- $\beta$ region for $\beta \approx 1.1$ (Fig. 2). The symbols for the APG cases are reported in Table I. Magenta symbols indicate the outer peak location for the ZPG cases from Ref. [32].

This can be traced back to the behavior of the ratio of boundary-layer thicknesses, i.e. $\delta^{*} / \delta_{99}$, which decreases for ZPGs, while it increases for APGs due to the relatively stronger increase of $\delta^{*}$ with pressure gradient $[26,35]$. In fact, the slightly increased trend observed for the APG case in the last two points may be connected with the decreased $\beta$ behavior of these last points (Fig. 2). Comparing the near-wall peak values with the evolution reported by Maciel et al. [27] it is observed that the inner-peak values are consistent with the proposed evolution of $\overline{u^{2}} / U_{\mathrm{ZS}}^{2}$ against $H_{12}$, which is represented in Fig. 9 from Maciel et al. [27]. Here, a comparison between $H_{12}$ and $\overline{u^{2}} / U_{\mathrm{ZS}}^{2}$ is not shown due to our limited $H_{12}$ range. As pointed out in the introduction of this section, the relationships analyzed by Maciel et al. [27] are obtained using the maximum value of $\overline{u^{2}} / U_{\mathrm{ZS}}^{2}$ without any distinction between inner-peak or outer-peak values. As a consequence, all the trends for $y_{\max }$ and $\bar{u}^{2}$ max reported in Ref. [27] display a discontinuity when plotted against $\beta$ (around $\beta \approx 3$ ) or an abrupt drop when plotted against the shape factor $H_{12}$. This inflection point in their figures indicates the point where the outer peak becomes dominant, i.e., the maximum location switches from the inner peak to the outer peak. Here, instead, the outer-peak evolution is analyzed separately, even when its value is not dominant over the inner peak. Analyzing the evolution of $\overline{u^{2}}$ max,outer $/ U_{\mathrm{ZS}}^{2}$ in Fig. 8(a), it appears that the values reported here for the outer peak are more consistent in order to describe the APG-peak effects since their evolution is closer to the trend 

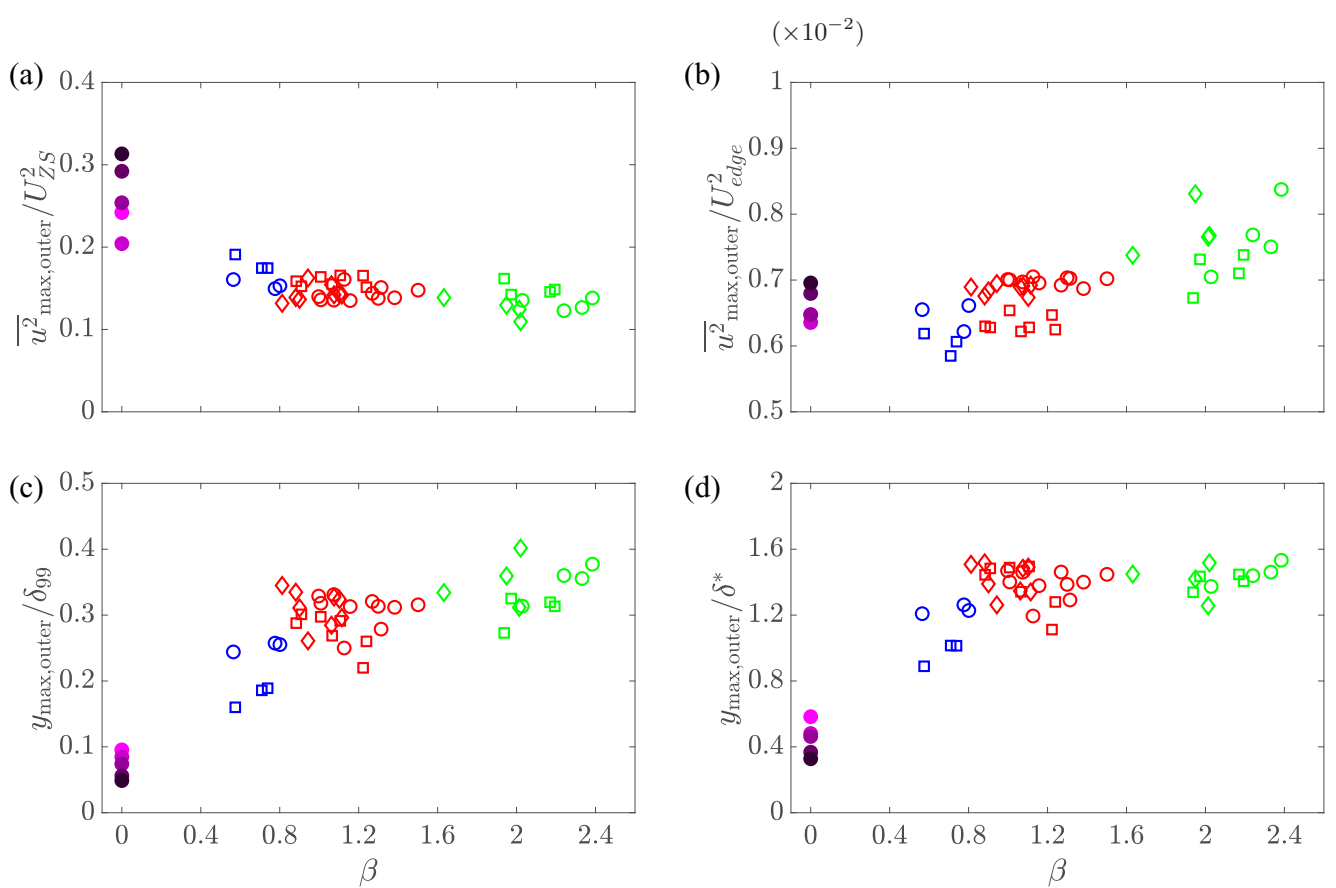

FIG. 9. Maximum value of the streamwise variance at the outer peak location normalized with (a) $U_{\mathrm{ZS}}$ and (b) $U_{\text {edge }}$ against $\beta$. Wall-normal position of the outer peak location normalized with (c) $\delta_{99}$ and (d) $\delta^{*}$ against $\beta$. The data shown correspond to the region $3.1 \mathrm{~m} \leqslant x \leqslant 4.4 \mathrm{~m}$. The colors and symbols for the APG cases are reported in Table I. Magenta symbols indicate the ZPG outer peak location and value for the ZPG cases from Ref. [32]. Color varies from light magenta to dark magenta with increasing Re.

reported in Maciel et al. [27] for their high- $\beta$ cases. In contrast, Fig. 8(b) shows the outer peak normalized with $U_{\text {edge }}$ showing a less clear trend for APG and ZPG cases.

Figures $8(\mathrm{c})$ and $8(\mathrm{~d})$ show $y_{\max , \text { outer }}$ scaled with $\delta_{99}$ and $\delta^{*}$, respectively. They tend to reach values of approximately 0.3 and 1.5 , respectively, but for $y_{\max , \text { outer }} / \delta_{99}$ the trend appears to be slightly decreasing with increasing Re, and, in contrast, for $y_{\max , \text { outer }} / \delta^{*}$ the trend seems to be approximately constant for the APG case. Figure 8 shows that a certain streamwise distance is required to reach an asymptotic value; this observation is connected to the results from Refs. [19,35] where it is reported that some streamwise development is needed to converge to "near-equilibrium" conditions for cases with non-constant- $\beta$ history. It has to be remarked that cases with variable $\beta$ but with a free-stream distribution given by a power law can reach the "near-equilibrium" state, i.e., self-similarity of the outer region in the mean profile in defect form at high $\mathrm{Re}$, as described in the classical theory by Townsend [56].

Figure 9 shows the evolution of the normalized outer peak $\overline{u^{2}}$ and of its location $y_{\text {max,outer }}$ scaled with $\delta_{99}$ and $\delta^{*}$ against $\beta$. Figure 9(a), which presents $\overline{u^{2}} / U_{\mathrm{ZS}}^{2}$, shows that the values of the outer peak reported slightly decrease with increasing $\beta$. On the other hand, Fig. 9(b) shows the opposite trend for $\overline{u^{2}} / U_{\text {edge }}^{2}$ with a more scattered distribution compared to Fig. 9(a). The evolution of $y_{\text {max }, \text { outer }} / \delta_{99}$ from Fig. 9(c) presents an increasing trend that complements the range of $\beta$ not considered in the study by Maciel et al. [27]. In the particular case of $\beta>1$ and high-Re the location of the outer peak is approximately $0.3 \delta_{99}$, which is in agreement with the observations in Ref. [15] and justifies the decision of choosing this value as a comparison for the ZPG spectra in Sec. III. For the case of $y_{\max , \text { outer }} / \delta^{*}$ shown in Fig. 9(d), the results confirm that for $\beta>1$ the location of the outer peak scales roughly with $\delta^{*}$ in our range of Re as predicted by Maciel et al. [27]. The observed 
small scatter is associated with Re effects as it is evidenced by the ZPG cases. For a more precise estimation, a complete characterization of the $y_{\max , \text { outer }}$ in terms of $\beta$ and Re would be necessary to take into account both effects. As a final remark, the comparison between $U_{\mathrm{ZS}}^{2}$ and $U_{\text {edge }}^{2}$ as velocity scaling parameters shows that, according to the results of Figs. 8 and 9, the ZagarolaSmits velocity scale appears to be a more adequate outer velocity scale as pointed out by Maciel et al. [27].

\section{CONCLUSIONS}

The difference between Reynolds-number and APG effects (in particular regarding the outer peak of streamwise fluctuations variance) is addressed via analysis of an experimental database of TBLs at high Reynolds number developing under APG and ZPG conditions, with the main goal of separating the effects of Reynolds number and pressure gradient. To this end, a novel method to identify the position of the outer peak has been introduced. The method is based on the empirical observation that the cross-over point between the small-scale variance under events of positive or negative large-scale fluctuations coincides with the outer-peak position. Since the method is based on an empirical criterion, it should be carefully validated and verified when applied to other data sets. Significant differences between the effects of APG and Reynolds number are identified:

(1) A scale-decomposition analysis highlights that the small-scale energy in APG TBLs is not universal, i.e. it depends on both Re and the APG magnitude. This is contrary to the well-established scaling of canonical ZPG TBLs. This has important consequences for the assumption of small-scale universality throughout the boundary layer, which is, e.g., made to correct for finite hot-wire spatialresolution effects, or in a broader context, for near-wall turbulence models.

(2) The same scale-decomposition analysis shows that the pressure gradient enhances the smallscale activity in the outer region, thus contrasting with the picture of high-Re ZPG, where the main effect of the Reynolds number is to mainly increase the large-scale energy.

(3) Our results indicate that, at sufficiently high Re, APG TBLs exhibit two large-scale spectral peaks in the outer region: one associated to high Reynolds numbers with $\lambda_{x} / \delta_{99} \approx 6$, which is also present in the outer region of high-Re ZPG TBLs [9]; and another related to the APG [15], with a streamwise wavelength of $\lambda_{x} / \delta_{99} \approx 3$.

(4) Regarding the streamwise variance profile, the wall-normal location of the outer peak arising for high enough Re in ZPG TBLs decreases with Re when normalized in outer units. On the other hand, the outer-peak location due to the APG appears to be independent of Re, and seems to depend only on the APG magnitude $\beta$.

The present paper contributes to answering the questions raised, among others, in Harun et al. [15] and Maciel et al. [27], who called for high-Reynolds-number APG TBL experiments to discern between Reynolds-number and APG effects on the scaling of the streamwise velocity fluctuations. Our results demonstrate that the differences between the two effects are indeed significant, and APG TBLs behave differently from high-Re ZPG TBLs. It would be very interesting to apply a similar analysis to well-resolved numerical simulations of boundary layers at sufficiently high Reynolds numbers with carefully controlled pressure gradients. In those cases, potentially an even clearer separation between APG and Re effects is possible, since no resolution effects are present, and more involved filter operations could be applied, including also the spanwise direction.

\section{ACKNOWLEDGMENTS}

C.S.V. acknowledges the financial support from Universidad Carlos III de Madrid within the program “Ayudas para la Movilidad del Programa Propio de Investigación.” R.Ö., R.V., and P.S. acknowledge the financial support from the Swedish Research Council (VR) and the Knut and Alice Wallenberg (KAW) Foundation as part of the Wallenberg Academy Fellow programme. C.S.V., 
S.D., and A.I. were partially supported by the Grant DPI2016-79401-R funded by the Spanish State Research Agency (SRA) and European Regional Development Fund (ERDF).

[1] N. Hutchins, T. B. Nickels, I. Marusic, and M. S. Chong, Hot-wire spatial resolution issues in wallbounded turbulence, J. Fluid Mech. 635, 103 (2009).

[2] G. Eitel-Amor, R. Örlü, and P. Schlatter, Simulation and validation of a spatially evolving turbulent boundary layer up to $\operatorname{Re}_{\theta}=8300$, Int. J. Heat Fluid Flow 47, 57 (2014).

[3] M. Samie, I. Marusic, N. Hutchins, M. K. Fu, Y. Fan, M. Hultmark, and A. J. Smits, Fully resolved measurements of turbulent boundary layer flows up to $\mathrm{Re}_{\tau}=20000$, J. Fluid Mech. 851, 391 (2018).

[4] R. Örlü and P. H. Alfredsson, Comment on the scaling of the near-wall streamwise variance peak in turbulent pipe flows, Exp. Fluids 54, 1431 (2013).

[5] C. E. Willert, J. Soria, M. Stanislas, J. Klinner, O. Amili, M. Eisfelder, C. Cuvier, G. Bellani, T. Fiorini, and A. Talamelli, Near-wall statistics of a turbulent pipe flow at shear Reynolds numbers up to 40000 , J. Fluid Mech. 826, R5 (2017).

[6] M. Lee and R. D. Moser, Direct numerical simulation of turbulent channel flow up to $R e_{\tau} \approx 5200$, J. Fluid Mech. 774, 395 (2015).

[7] J. C. D. Alamo and J. Jiménez, Spectra of the very large anisotropic scales in turbulent channels, Phys. Fluids 15, L41 (2003).

[8] I. Marusic and G. J. Kunkel, Streamwise turbulence intensity formulation for flat-plate boundary layers, Phys. Fluids 15, 2461 (2003).

[9] R. Mathis, N. Hutchins, and I. Marusic, Large-scale amplitude modulation of the small-scale structures in turbulent boundary layers, J. Fluid Mech. 628, 311 (2009).

[10] I. Marusic, R. Mathis, and N. Hutchins, High Reynolds number effects in wall turbulence, Int. J. Heat Fluid Flow 31, 418 (2010).

[11] R. Örlü and P. Schlatter, On the fluctuating wall shear stress in zero pressure-gradient turbulent boundary layer flows, Phys. Fluids 23, 021704 (2011).

[12] P. H. Alfredsson, A. Segalini, and R. Örlü, A new scaling for the streamwise turbulence intensity in wall-bounded turbulent flows and what it tells us about the "outer" peak, Phys. Fluids 23, 041702 (2011).

[13] M. Hultmark, M. Vallikivi, S. C. C. Bailey, and A. J. Smits, Turbulent Pipe Flow at Extreme Reynolds Numbers, Phys. Rev. Lett. 108, 094501 (2012).

[14] M. Vallikivi, M. Hultmark, and A. J. Smits, Turbulent boundary layer statistics at very high Reynolds number, J. Fluid Mech. 779, 371 (2015).

[15] Z. Harun, J. P. Monty, R. Mathis, and I. Marusic, Pressure gradient effects on the large-scale structure of turbulent boundary layers, J. Fluid Mech. 715, 477 (2013).

[16] P. E. Skåre and P. Krogstad, A turbulent equilibrium boundary layer near separation, J. Fluid Mech. 272, 319 (1994).

[17] C. Sanmiguel Vila, R. Örlü, R. Vinuesa, P. Schlatter, A. Ianiro, and S. Discetti, Adverse-pressure-gradient effects on turbulent boundary layers: Statistics and flow-field organization, Flow Turbul. Combust. 99, 589 (2017).

[18] I. Marušić and A. E. Perry, A wall-wake model for the turbulence structure of boundary layers. Part 2. Further experimental support, J. Fluid Mech. 298, 389 (1995).

[19] A. Bobke, R. Vinuesa, R. Örlü, and P. Schlatter, History effects and near equilibrium in adverse-pressuregradient turbulent boundary layers, J. Fluid Mech. 820, 667 (2017).

[20] P. R. Spalart and J. H. Watmuff, Experimental and numerical study of a turbulent boundary layer with pressure gradients, J. Fluid Mech. 249, 337 (1993).

[21] J. H. Lee, Large-scale motions in turbulent boundary layers subjected to adverse pressure gradients, J. Fluid Mech. 810, 323 (2017). 
[22] V. Kitsios, C. Atkinson, J. Sillero, G. Borrell, A. Gungor, and J. Jiménez, Direct numerical simulation of a self-similar adverse pressure gradient turbulent boundary layer, Int. J. Heat Fluid Flow 61, 129 (2016).

[23] R. Vinuesa, P. S. Negi, M. Atzori, A. Hanifi, D. S. Henningson, and P. Schlatter, Turbulent boundary layers around wing sections up to $R e_{c}=1,000$, 000, Int. J. Heat Fluid Flow 72, 86 (2018).

[24] Y. Maciel, K. Rossignol, and J. Lemay, A study of a turbulent boundary layer in stalled-airfoil-type flow conditions, Exp. Fluids 41, 573 (2006).

[25] V. Kitsios, A. Sekimoto, C. Atkinson, J. A. Sillero, G. Borrell, A. G. Gungor, J. Jiménez, and J. Soria, Direct numerical simulation of a self-similar adverse pressure gradient turbulent boundary layer at the verge of separation, J. Fluid Mech. 829, 392 (2017).

[26] R. Vinuesa, R. Örlü, C. Sanmiguel Vila, A. Ianiro, S. Discetti, and P. Schlatter, Revisiting history effects in adverse-pressure-gradient turbulent boundary layers, Flow Turbul. Combust. 99, 565 (2017).

[27] Y. Maciel, T. Wei, A. G. Gungor, and M. P. Simens, Outer scales and parameters of adverse-pressuregradient turbulent boundary layers, J. Fluid Mech. 844, 5 (2018).

[28] T. Wei and Y. Maciel, Derivation of Zagarola-Smits scaling in zero-pressure-gradient turbulent boundary layers, Phys. Rev. Fluids 3, 012601 (2018).

[29] Y. Maciel, K. Rossignol, and J. Lemay, Self-similarity in the outer region of adverse-pressure-gradient turbulent boundary layers, AIAA J. 44, 2450 (2006).

[30] N. Hutchins and I. Marusic, Large-scale influences in near-wall turbulence, Philos. Trans. R. Soc. A. 365, 647 (2007).

[31] N. Hutchins and I. Marusic, Evidence of very long meandering features in the logarithmic region of turbulent boundary layers, J. Fluid Mech. 579, 1 (2007).

[32] R. Örlü, Experimental studies in jet flows and zero pressure-gradient turbulent boundary layers, Ph.D. thesis, KTH Royal Institute of Technology, Stockholm, Sweden, 2009.

[33] R. Örlü and P. Schlatter, Comparison of experiments and simulations for zero pressure gradient turbulent boundary layers at moderate Reynolds numbers, Exp. Fluids 54, 1547 (2013).

[34] A. J. Smits, J. P. Monty, M. Hultmark, S. C. C. Bailey, N. Hutchins, and I. Marusic, Spatial resolution correction for wall-bounded turbulence measurements, J. Fluid Mech. 676, 41 (2011).

[35] C. Sanmiguel Vila, R. Vinuesa, S. Discetti, A. Ianiro, P. Schlatter, and R. Örlü, Experimental realization of near-equilibrium adverse-pressure-gradient turbulent boundary layers, Exp. Therm. Fluid Sci. 112, 109975 (2020).

[36] J. M. Österlund, Experimental studies of zero pressure-gradient turbulent boundary layer flow, Ph.D. thesis, KTH Royal Institute of Technology, Stockholm, Sweden, 1999.

[37] B. Lindgren and A. V. Johansson, Evaluation of the flow quality in the MTL wind-tunnel, Tech. Rep. TRITA-MEK 2002:13, KTH Royal Institute of Technology, Stockholm, Sweden, 2002.

[38] C. Sanmiguel Vila, R. Vinuesa, S. Discetti, A. Ianiro, P. Schlatter, and R. Örlü, On the identification of well-behaved turbulent boundary layers, J. Fluid Mech. 822, 109 (2017).

[39] R. Örlü and P. H. Alfredsson, On spatial resolution issues related to time-averaged quantities using hotwire anemometry, Exp. Fluids 49, 101 (2010).

[40] K. A. Chauhan, P. A. Monkewitz, and H. M. Nagib, Criteria for assessing experiments in zero pressure gradient boundary layers, Fluid Dyn. Res. 41, 021404 (2009).

[41] R. Vinuesa, A. Bobke, R. Örlü, and P. Schlatter, On determining characteristic length scales in pressuregradient turbulent boundary layers, Phys. Fluids 28, 055101 (2016).

[42] J. C. del Álamo and J. Jiménez, Estimation of turbulent convection velocities and corrections to Taylor's approximation, J. Fluid Mech. 640, 5 (2009).

[43] A. Dróżdż and W. Elsner, Amplitude modulation and its relation to streamwise convection velocity, Int. J. Heat Fluid Flow 63, 67 (2017).

[44] J. P. Monty, Z. Harun, and I. Marusic, A parametric study of adverse pressure gradient turbulent boundary layers, Int. J. Heat Fluid Flow 32, 575 (2011).

[45] E. Dogan, R. Örlü, D. Gatti, R. Vinuesa, and P. Schlatter, Quantification of amplitude modulation in wall-bounded turbulence, Fluid Dyn. Res 51, 011408 (2019).

[46] P. Moin, Revisiting Taylor's hypothesis, J. Fluid Mech. 640, 1 (2009). 
[47] I. Marusic, B. J. McKeon, P. A. Monkewitz, H. M. Nagib, A. J. Smits, and K. R. Sreenivasan, Wallbounded turbulent flows at high Reynolds numbers: Recent advances and key issues, Phys. Fluids 22, 065103 (2010).

[48] J. H. Lee, Kevin, J. P. Monty, and N. Hutchins, Validating under-resolved turbulence intensities for PIV experiments in canonical wall-bounded turbulence, Exp. Fluids 57, 129 (2016).

[49] R. Deshpande, J. P. Monty, and I. Marusic, Correcting hotwire misalignment errors for wall-bounded turbulence measurements, in Proceedings of the International Symposium on Turbulence \& Shear Flow Phenomena (TSFP-11), Southampton, United Kingdom (2019).

[50] C. Chin, N. Hutchins, A. Ooi, and I. Marusic, Spatial resolution correction for hot-wire anemometry in wall turbulence, Exp. Fluids 50, 1443 (2011).

[51] R. Deshpande, J. P. Monty, and I. Marusic, A scheme to correct the influence of calibration misalignment for cross-wire probes in turbulent shear flows, Exp. Fluids 61, 90 (2020).

[52] A. Segalini, G. Bellani, G. Sardina, L. Brandt, and E. A. Variano, Corrections for one- and two-point statistics measured with coarse-resolution particle image velocimetry, Exp. Fluids 55, 1739 (2014).

[53] B. Ganapathisubramani, N. Hutchins, J. P. Monty, D. Chung, and I. Marusic, Amplitude and frequency modulation in wall turbulence, J. Fluid Mech. 712, 61 (2012).

[54] M. Yoon, J. Hwang, and H. J. Sung, Contribution of large-scale motions to the skin friction in a moderate adverse pressure gradient turbulent boundary layer, J. Fluid Mech. 848, 288 (2018).

[55] R. Mathis, I. Marusic, N. Hutchins, and K. Sreenivasan, The relationship between the velocity skewness and the amplitude modulation of the small scale by the large scale in turbulent boundary layers, Phys. Fluids 23, 121702 (2011).

[56] A. Townsend, The properties of equilibrium boundary layers, J. Fluid Mech. 1, 561 (1956). 